Vol. 43 (1991) [1-17]

\title{
VARIATIONAL AND NUMERICAL METHODS FOR SYMMETRIC MATRIX PENCILS
}

\author{
Peter lancaster and Qiang Ye
}

\begin{abstract}
A review is presented of some recent advances in variational and numerical methods for symmetric matrix pencils $\lambda A-B$ in which $A$ is nonsingular, $A$ and $B$ are hermitian, but neither is definite. The topics covered include minimax and $\max$ imin characterisations of eigenvalues, perturbation by semidefinite matrices and interlacing properties of real eigenvalues, Rayleigh quotient algorithms and their convergence properties, Rayleigh-Ritz methods employing Krylov subspaces, and a generalised Lanczos algorithm.
\end{abstract}

\section{INTRODUCTION}

Pencils of matrices $\lambda A-B$ where $A$ and $B$ are both hermitian (or real symmetric), and $\lambda$ is a real or complex parameter occur in a wide variety of applications of mathematics. The case in which $A$ (or $B$ ) is positive definite is well-understood although the algorithm development still goes on (see [1], for example). When both $A$ and $B$ are indefinite the spectral properties of the pencil can be considerably more complicated, but such pencils arise naturally in system theory, factorisation of matrix functions, vibration problems, and the analysis of more general hermitian and sympletic transformations (see references $[2,9,10,15]$ and many others), and so they are now a focus of mathematical analysis and algorithm development (see [7, 25, 34], for example). It is therefore timely to present a survey of some recent extensions of classical variational methods and dependent numerical methods for such problems, and that is our objective in this paper.

We say that $\lambda_{0}$ is an eigenvalue and $x$ a corresponding eigenvector of a pencil $\lambda A-B$ if $\left(\lambda_{0} A-B\right) x=0$ and $x \neq 0$. We suppose that $A$ and $B$ are hermitian and that $A$ is nonsingular but not necessarily definite. When $A$ is positive definite, the algebraic problem is equivalent to the eigenvalue problem of a single hermitian or real symmetric matrix. But when $A$ is indefinite, the problem is significantly harder. Indeed, for the real case, it is known that every (generally non-symmetric) real regular matrix pencil

Received 15 January 1990

Research supported in part by a grant from the Nature Science and Engineering Research Council of Canads

Copyright Clearance Centre, Inc. Serial-fee code: 0004-9729/91 \$A2.00+0.00. 
(when $\operatorname{det}(\lambda A-B)$ is not identically zero) is equivalent to a real symmetric matrix pencil (see Theorem 4 of [16]). It is our intention to survey some recent progress that shows to what extent the classical variational results and related numerical methods for single symmetric matrices have been carried over to our symmetric pencil case.

We mention that some arguments can be extended to the infinite dimensional case by introducing an indefinite metric defined by $A$. But we will concentrate on the finite dimensional case as we believe that several essential insights are revealed in this context, and because this has to be understood for computational purposes.

We give a survey of results that are presently clear, and without proofs in most cases. The details appear in independent works (see $[17,18,36])$. Our purpose here is to give a birds-eye view of the emerging theory and algorithms.

Some preliminary ideas, including canonical forms for hermitian matrix pairs under congruence, are presented first of all. Then Section 3 contains generalised min-max and max-min characterisations of eigenvalues for diagonable hermitian pencils, and comparison with early results of Phillips. These results are used in Section 4 to derive the effects of positive perturbations on the eigenvalues of such pencils. The topic of numerical algorithms is broached in Section 5 with discussion of local and global convergence properties of generalised Rayleigh quotient algorithms. The global results are confined to definite pencils (defined in Section 2). The next topic is Rayleigh-Ritz projection methods for definite pencils. Extensions of classical theory are presented which admit eigenvalue estimates and finally, the extension of Lanczos tridiagonalisation algorithm to pencil problems.

The reader may like to keep in mind four significant problems classes in increasing degrees of generality.

1. Hermitian pencils with $A>0$ (the "classical" case).

2. Definite pencils (there exist real numbers $\alpha$ and $\beta$ such that $\alpha A+\beta B>$ 0).

3. Diagonable pencils with real spectrum.

4. Hermitian pencils with $A$ nonsingular.

The results summarised in this paper refer mainly to classes 2 and 3 . Some generalisations to the fourth class are also known. See [20] for the basic minimax theory, for example.

\section{Prototypes and Preliminaries}

Variational characterisations of eigenvalues for hermitian matrices extend easily to pencils $\lambda A-B$ where $A^{*}=A, B^{*}=B$ and $A>0$. Indeed, by introducing a scalar product defined by $\langle x, y\rangle=y^{*} A x$, the pencil eigenvalue problem is found to be 
equivalent to the eigenvalue problem for a single transformation that is self-adjoint with respect to $\langle$,$\rangle .$

The Courant-Fischer characterisation of eigenvalues for $\lambda A-B$ with $A>0$ takes the following form $([3,8]$; see also [19]):

Theorem 2.1 . Let $S_{j}(1 \leqslant j \leqslant n)$ denote an arbitrary $(n-j+1)$-dimensional subspace of $C^{n}$, and $\lambda_{1} \leqslant \ldots \leqslant \lambda_{n}$ be eigenvalues of an hermitian pencil with $A$ positive definite. Then, for $j=1, \ldots, n$,

$$
\lambda_{j}=\max _{S_{j}} \min _{\substack{x \in S_{j} \\ x \neq 0}} \frac{x^{*} B x}{x^{*} A x},
$$

and

$$
\lambda_{n-j+1}=\min _{S_{j}} \max _{\substack{x \in S_{j} \\ x \neq 0}} \frac{x^{*} B x}{x^{*} A x} .
$$

Another point of view on the eigenvalue problem for matrix pencils concerns the simultaneous reduction of the quadratic forms associated with $A$ and $B$. Again, if $A>$ 0 , it is well known that $A$ and $B$ can be simultaneously diagonalised by congruence. That is, there is a nonsingular matrix $X$ such that

$$
X^{*} A X=I, \quad X^{*} B X=\Lambda
$$

where $\Lambda$ is the diagonal matrix of eigenvalues of $\lambda A-B$.

Our objective is to present a generalisation of Courant-Fischer theory to indefinite pencils (where we replace the condition $A>0$ by $\operatorname{det} A \neq 0$ ), together with its implications for perturbation theory, the development of the Rayleigh-Ritz method, and some relevant numerical methods. However, these generalisations will be approached via the corresponding reduction of two quadratic forms, neither of which is definite.

The critical initial step is once more to introduce a scalar product defined by $\langle x, y\rangle=y^{*} A x$ even though it will now be indefinite (the invertibility of $A$ ensures that $\langle$,$\rangle is nondegenerate, however). The canonical form for the pencil under congruence$ now depends strongly on the Jordan canonical form for $A^{-1} B$. Let us state the complete theorem. This has a history going back to Weierstrass [35], and a complete proof in this form can be found in [9]. The set of the eigenvalues of a square matrix $M$ is called the spectrum of $M$, and denoted by $\sigma(M)$.

TheOREM 2.2. If $A, B$ are hermitian matrices with $A$ invertible then there is a nonsingular matrix $X$ such that

$$
X^{*} A X=P_{\varepsilon, J}, \quad X^{*} B X=P_{e, J} J
$$

where $J=J_{c} \oplus J_{r} \oplus \bar{J}_{c}$ is the Jordan matrix of $A^{-1} B$, with $\sigma\left(J_{r}\right)$ real and $\lambda \in \sigma\left(J_{c}\right)$ implies $\operatorname{Im} \lambda>0$, and $P_{e, J}$ is a canonical matrix defined by $J$ and a sign characteristic 
$\varepsilon$ in the following way: we have $P_{e, J}=\left[\begin{array}{ccc}0 & 0 & P_{c} \\ 0 & P_{r} & 0 \\ P_{c} & 0 & 0\end{array}\right]$ and if $J_{c}, J_{r}$ are expressed in terms of Jordan blocks by $J_{c}=\operatorname{diag}\left[J_{1}, \ldots, J_{k}\right], J_{r}=\operatorname{diag}\left[J_{k+1}, \ldots, J_{\ell}\right]$, then $P_{c}$, $P_{r}$ have a corresponding block-diagonal structure:

$$
P_{c}=\operatorname{diag}\left[P_{1}, \ldots, P_{k}\right], \quad P_{r}=\operatorname{diag}\left[\varepsilon_{k+1} P_{k+1}, \ldots, \varepsilon_{\ell} P_{\ell}\right]
$$

where $P_{i}$ is a s.i.p. matrix, that is, $P_{i}=\left[\begin{array}{c}\cdot \\ .\end{array}\right]$ and $\varepsilon_{k+1}, \ldots, \varepsilon_{\ell}$ are each equal to +1 or -1 . The ordered set $\varepsilon=\left\{\varepsilon_{k+1}, \ldots, \varepsilon_{\ell}\right\}$, which is called a sign characteristic of the pair $A, B$, is uniquely determined to within permutation of signs corresponding to equal blocks $J_{i}$.

In a natural way, we may also associate a sign characteristic with each real eigenvalue of $\lambda A-B$. It is the set of +1 's and -1 's associated with the Jordan blocks of the eigenvalue in question. In particular, an eigenvalue of mixed type may be viewed as a superposition of eigenvalues of positive and negative types. When $\lambda A-B$ is diagonable (that is, when $A^{-1} B$ is diagonable) the number of entries in the sign characteristic of a real eigenvalue is just the multiplicity of the eigenvalue.

We say an hermitian matrix pencil $\lambda A-B$ is definite if there exist $\alpha, \beta \in \mathbf{R}$ such that $\alpha A+\beta B$ is positive definite. The notion of definite pencils has played an important part in the literature. One of the most important properties of this kind of pencil is its geometric characterisation (see [5] and the references therein), that is, that an hermitian pencil $\lambda A-B$ is definite if and only if

$$
c(A, B):=\inf \left\{\left|x^{*}(A+i B) x\right|>0:\|x\|=1\right\}>0 .
$$

Furthermore, a definite pencil is diagonable and all its eigenvalues are real. On the other hand, a diagonable pencil with all eigenvalues real is not necessarily definite. Investigation of this question leads to the following spectral characterisation of definite pencils (see [17]).

Theorem 2.3. An hermitian pencil $\lambda A-B$ with $A$ invertible is definite if and only if it is diagonable, has all eigenvalues real, and the eigenvalues of positive type and the eigenvalues of negative type are separated.

Here, "separated" has the natural meaning; either all eigenvalues of positive type exceed all eigenvalues of negative type, or vice versa. A connection is made between the indefinite and classical cases in the use of subspaces that are positive (nonnegative, 
et cetera) with respect to $A$. Thus a subspace $S$ is strictly $A$-positive ( $A$-negative) if $x^{*} A x>0$ ( $<0$, respectively) for all nonzero $x$ in $S$, and it is said to be maximal if it is not a proper subspace of a strictly $A$-positive ( $A$-negative) subspace.

\section{Minimax Principle}

The classical minimax principles (Theorem 2.1) are aimed at conservative vibrating system problems. A number of generalisations of minimax principles to non-conservative systems have been made in the last decades (for example $[6,28,33]$ ). For definite matrix pencils, Stewart [30] has a generalisation in terms of certain angles. Textorius [32] discussed self-adjoint operators in an indefinite scalar product space, and his work extends that of Phillips [27], whose definitions are presented below (specialised to finite dimensions) to enable direct comparisons.

We are going to present a direct generalisation of Theorem 2.1 (see [17]). Our approach requires that $\lambda A-B$ has real eigenvalues (which is not necessarily the case) and that they have certain special properties.

THEOREM 3.1. Let $\lambda A-B$ be a diagonable hermitian matrix pencil with $A$ invertible and all eigenvalues real. Suppose that $\lambda_{1} \leqslant \ldots \leqslant \lambda_{r}<\lambda_{r+1} \leqslant \ldots \leqslant \lambda_{k}$ are eigenvalues of negative type, $\lambda_{k+1} \leqslant \ldots \leqslant \lambda_{s-1}<\lambda_{k} \leqslant \ldots \leqslant \lambda_{n}$ are eigenvalues of positive type and $\lambda_{r}<\left\{\lambda_{r+1}, \ldots, \lambda_{k}, \lambda_{k+1}, \ldots, \lambda_{\text {s-1 }}\right\}<\lambda_{\text {s. }}$. Then

$$
\begin{gathered}
\lambda_{k+j}=\sup _{\operatorname{dim} S_{j=n-j+1}} \inf _{\substack{x \in S_{j} \\
x^{*} A x>0}} \frac{x^{*} B x}{x^{*} A x}, \quad \text { for } k+j \geqslant s, \\
\lambda_{k-j+1}=\inf _{\operatorname{dim} S_{j=n-j+1}} \sup _{\substack{x \in S_{j} \\
x^{*} A z<0}} \frac{x^{*} B x}{x^{*} A x}, \quad \text { for } k-j+1 \leqslant r .
\end{gathered}
$$

As a special case, the following corollary gives a minimax characterisation for a definite matrix pencil

Corollary 3.2. Let $\lambda A-B$ be a diagonable hermitian matrix pencil with $A$ invertible and all eigenvalues real. Suppose $\lambda_{1} \leqslant \ldots \leqslant \lambda_{k}<\lambda_{k+1} \leqslant \ldots \leqslant \lambda_{n}$ are eigenvalues with $\lambda_{1}, \ldots, \lambda_{k}$ negative type, and $\lambda_{k+1}, \ldots, \lambda_{n}$ positive type, then

$$
\begin{aligned}
& \lambda_{i}=\inf _{\operatorname{dim} S_{i=n-k+i}} \sup _{\substack{x \in S_{i} \\
x^{*} A x<0}} \frac{x^{*} B x}{x^{*} A x}, \quad \text { for } i=1, \ldots, k . \\
& \lambda_{j}=\operatorname{dim}_{S_{j}=n+k+1-i} \operatorname{iuf}_{\substack{x \in S_{j} \\
z^{*} A x>0}} \frac{x^{*} B x}{x^{*} A x}, \quad \text { for } j=k+1, \ldots, n .
\end{aligned}
$$


In particular,

$$
\lambda_{k}=\max _{x^{*} A x<0} \frac{x^{*} B x}{x^{*} A x}, \quad \lambda_{k+1}=\min _{x^{*} A x>0} \frac{x^{*} B x}{x^{*} A x} .
$$

By considering $\lambda A+B$, we can obtain corresponding results for the case that eigenvalues of positive type are less than eigenvalues of negative type.

For an hermitian matrix $H$, we consider the pencil $\lambda I-H$. In this case all eigenvalues are of positive type. So, in a trivial way, we can regard the pencil $\lambda I-H$ as having negative type eigenvalues less than positive type eigenvalues, or vice versa. Then the corollary will apply and give both the max-min and min-max characterisations of the classical theory. In contrast to this, the above results show that when eigenvalues of both types occur, our max-min and min-max characterisations apply only to eigenvalues of positive and negative types, respectively.

We remark that if $\lambda A-B$ is a real symmetric pencil with all eigenvalues real, then all discussions can be completed within the real space $\mathbf{R}^{\boldsymbol{n}}$ and we have corresponding results of the same form with all subspaces $S_{i} \subset \mathbf{R}^{n}$.

The only comparable generalised minimax criterion that we know in this direction is that of Phillips [27], whose results are established in the context of a Hilbert space with an indefinite metric. We mention that Theorem 3.1 can also be generalised to the infinite dimensional case, provided a suitable canonical form for operator pencils is known. For comparison we now state Phillips' theorem formulated in the matrix context.

THEOREM 3.3. Let $\lambda A-B$ be an hermitian pencil with $A$ invertible and $B$ positive definite, and $\gamma_{1} \geqslant \ldots \geqslant \gamma_{k}>0>\kappa_{l} \geqslant \ldots \geqslant \kappa_{1}$ be its eigenvalues. Let $N$ and $P$ denote strictly $A$-negative and strictly $A$-positive maximal subspaces, $S$ be an arbitrary subspace of dimension $i-1$. Then

$$
\begin{gathered}
\kappa_{i}=\sup _{N} \sup _{S \subset N} \inf _{\substack{x \in N \\
x^{*} A S=0}} \frac{x^{*} B x}{x^{*} A x}, \\
\gamma_{i}=\inf _{P} \inf _{S \subset P} \sup _{\substack{x \in P \\
z^{*} A S=0}} \frac{x^{*} B x}{x^{*} A x} .
\end{gathered}
$$

The assumption that $B$ is positive definite is equivalent to assuming that $\lambda A-B$ is definite (the original assumption in [27] is that $A^{-1} B$ is $A$-positive). In this case $\gamma_{i}$ are positive eigenvalues of positive type, and $\kappa_{i}$ are negative eigenvalues of negative type.

Let us make some brief comparisons between Theorems 3.1 and 3.3. First Theorem 3.3 is confined to pencils that are definite, then a translation of the parameter will 
produce a positive definite $B$ matrix. When both results apply, that is, comparing Corollary 3.2 with Theorem 3.3, the "double" inf-sup or sup-inf characterisation are more easily applied than the tripled process of Theorem 3.3. Also, Corollary 3.2 leads to the simple characterisations of equations (3.1), which do not follow directly from Theorem 3.3. There is also an interesting contrast in the ordering of the sup-inf steps in the two descriptions.

\section{Positive perturbations}

It is well known that the classical minimax principle implies that if an hermitian matrix is pert urbed by a positive semidefinite matrix, the eigenvalues can only increase, moreover, an interlacing property holds depending on the rank of the perturbation matrix. We pose a similar question for matrix pencils; that is, given a symmetric pencil $\lambda A-B$, how are eigenvalues of $\lambda A-(B+K)$ related to those of $\lambda A-B$ for a positive definite $K$. In Section 3.3.1 of [0] this question was investigated in the setting of the inner product space defined by $A$ and local perturbation results were established. It is shown there that if a simple real eigenvalue has multiplicity $r$ with $r=r_{+}+r_{-}$, and $r_{+}$' eigenvalues are of positive type and $r_{-}$are of negative type, then a positive definite perturbation of $B$ causes $r_{+}$eigenvalues to increase and $r_{-}$to decrease. This behaviour is confirmed here for the case of definite pencils (although Theorem 2.3 shows that eigenvalues of mixed type cannot arise in this case).

We consider a definite pencil $\lambda A-B$ with $A$ invertible and $\alpha A+B>0$ for some $\alpha$. In this case, the eigenvalues of positive type are greater than those of negative type. Suppose $\lambda_{1} \leqslant \lambda_{2} \leqslant \ldots \leqslant \lambda_{k}<\lambda_{k+1} \leqslant \ldots \leqslant \lambda_{n}$ are eigenvalues with $\lambda_{1}, \lambda_{2}, \ldots, \lambda_{k}$ negative type and $\lambda_{k+1}, \ldots, \lambda_{n}$ positive type. Clearly, the pencil $\lambda A-(B+K)$ with $K \geqslant 0$ is a definite pencil because $\alpha A+(B+K)>0$. Indeed, $K$ can be any matrix for which $\alpha A+(B+K)>0$. Since the number of positive (or negative) type eigenvalues is the number of the positive (negative, respectively) eigenvalues of $A$, we have that $\lambda A-(B+K)$ has $k$ negative type eigenvalues, $n-k$ positive type eigenvalues, and positive type eigenvalues must be greater than negative type eigenvalues. We denote them as $\mu_{1} \leqslant \mu_{2} \leqslant \ldots \leqslant \mu_{k}<\mu_{k+1} \leqslant \ldots \leqslant \mu_{n}$. Then Corollary 3.2 applies to both $\lambda_{i}$ and $\mu_{i}$. We write down the part for positive type eigenvalues as follows:

$$
\lambda_{j}=\sup _{\operatorname{dim} S_{j}=n+k+1-j} \inf _{\substack{x \in \mathcal{S}_{j} \\ x^{*} A x>0}} \frac{x^{*} B x}{x^{*} A x},
$$

and

$$
\mu_{j}=\sup _{\operatorname{dim} S_{j}=n+k+1-j} \inf _{\substack{x \in \mathcal{S}_{j} \\ x^{*} A x>0}}\left(\frac{x^{*} B x}{x^{*} A x}+\frac{x^{*} K x}{x^{*} A x}\right)
$$

for $j=k+1, \ldots, n$. Immediately we obtain the following monotonicity theorem. As 
noted above, the main idea is that, under the positive perturbation real eigenvalues of positive and negative type remain real and increase and decrease, respectively.

Theorem 4.1. Let $\lambda A-B$ be a definite pencil with $A$ invertible. Suppose $\lambda_{1} \leqslant \lambda_{2} \leqslant \ldots \leqslant \lambda_{k}<\lambda_{k+1} \leqslant \ldots \leqslant \lambda_{n}$ are eigenvalues with $\lambda_{1}, \lambda_{2}, \ldots, \lambda_{k}$ of negative type and $\lambda_{k+1}, \ldots, \lambda_{n}$ positive type. Then for a positive semi-definite matrix $K$, $\lambda A-(B+K)$ is definite too and has eigenvalues $\mu_{1} \leqslant \mu_{2} \leqslant \ldots \leqslant \mu_{k}<\mu_{k+1} \leqslant \ldots \leqslant \mu_{n}$ with $\mu_{1}, \mu_{2}, \ldots, \mu_{k}$ negative type and $\mu_{k+1}, \ldots, \mu_{n}$ positive type. Moreover,

and

$$
\mu_{i}-\sigma_{-1} \leqslant \lambda_{i}, \quad \text { for } i=1, \ldots k
$$

$$
\mu_{j} \geqslant \lambda_{j}+\sigma_{1}, \quad \text { for } j=k+1, \ldots n \text {. }
$$

where $\sigma_{1}$ is the smallest non-negative eigenvalue of $\lambda A-K$ and $\sigma_{-1}$ is the biggest non-positive eigenvalue of $\lambda A-K$.

Now, we suppose $K$ has rank 1 with $K=u u^{*}$. From the proof of Theorem 3.1, we have (see [17])

$$
\mu_{j}=\inf _{\substack{x \in T_{j} \\ x^{*} A x>0}}\left(\frac{x^{*} B x}{x^{*} A x}+\frac{x^{*} K x}{x^{*} A x}\right)
$$

where $T_{j}=\operatorname{span}\left\{e_{1}, \ldots, e_{k}, e_{j}, \ldots, e_{n}\right\}$ with $e_{i}$ the eigenvector corresponding to $\mu_{i}$. Then $\operatorname{dim}\left(T_{j} \cap\{u\}^{\perp}\right) \geqslant n+k-j$. Thus there exists a subspace $T_{0} \subset T_{j} \cap\{u\}^{\perp} \subset T_{j}$ with $\operatorname{dim} T_{0}=n+k-j$. Hence

$$
\begin{aligned}
\mu_{j} & \leqslant \inf _{\substack{x \in T_{0} \\
x^{*} A x>0}}\left(\frac{x^{*} B x}{x^{*} A x}+\frac{x^{*} K x}{x^{*} A x}\right) \\
& =\inf _{\substack{x \in T_{0} \\
x^{*} A x>0}} \frac{x^{*} B x}{x^{*} A x} \leqslant \lambda_{j+1} .
\end{aligned}
$$

So, we obtain the interlacing property:

THEOREM 4.2. Under the assumption of Theorem 4.1 and $\operatorname{rank}(K)=1$, we have

and

$$
\lambda_{i-1} \leqslant \mu_{i}, \quad \text { for } i=2, \ldots, k,
$$

$$
\mu_{j} \leqslant \lambda_{j+1}, \quad \text { for } j=k+1, \ldots, n-1 .
$$

Obviously, from the theorems, we can obtain a more general form with a rank $r$ positive semidefinite perturbation $K$ in the form

and

$$
\begin{array}{ll}
\lambda_{i-r} \leqslant \mu_{i} \leqslant \lambda_{i}, & \text { for } i=1, \ldots, k \\
\lambda_{j} \leqslant \mu_{j} \leqslant \lambda_{j+r}, & \text { for } j=k+1, \ldots, n,
\end{array}
$$


where we denote $\lambda_{i}=-\infty$ for $i \leqslant 0$ and $\lambda_{j}=+\infty$ for $j \geqslant n+1$.

By using a more general minimax theorem (see [20]) similar results can be established for more general hermitian pencils rather than just definite pencils. However, we have confined attention to definite pencils here for clear presentation of the idea and the details of this work will appear in an independent paper.

\section{RAYLEIGH QUOTIENT ITERATION}

The Rayleigh quotient iteration is a method for finding eigenvalues by using inverse iteration together with the Rayleigh quotient shift. Its local cubic convergence and global convergence properties make it a competitive method when only a few eigenvalues of a symmetric matrix are needed. The discussion of local convergence for a hermitian matrix was started in [31] and [4] and was analysed rigorously and in detail in Ostrowski [21]. In his series of papers, Ostrowski also generalised the analysis to admit non-hermitian matrices, and in Lancaster [14], its generalisation to nonlinear eigenvalue problems was discussed. Then came the proof of global convergence for hermitian matrices in Kahan [11], Parlett and Kahan [26], and its generalisation to normal matrices in Parlett [24]. Also, the corresponding results for symmetric matrix pencils $\lambda A-B$ with $A$ positive definite can be found in Parlett [23]. But there has been no discussion for the case in which $A$ is indefinite; the topic of this review.

We first observe that the Rayleigh quotient $\rho(x)=\frac{x^{*} B x}{x^{*} A x}$ is defined only for $x$ with $x^{*} A x \neq 0$. Then the following Rayleigh quotient algorithm is a natural extension of the classical case. We use $\|\cdot\|$ to denote any vector norm on $\mathbf{C}^{n}$.

ALGORITHM 5.1. Pick a starting vector $x_{0} \in C^{n}$ with $\left\|x_{0}\right\|=1$ and $x_{0}^{*} A x_{0}>0$ (or $x_{0}^{*} A x_{0}<0$ ). Then for $k=0,1,2, \ldots$

(i) if $x_{k}^{*} A x_{k}=0$, stop; otherwise

(ii) form $\rho_{k}=\rho\left(x_{k}\right)=\frac{x_{k}^{*} B x_{k}}{x_{k}^{*} A x_{k}}$;

(iii) if $\rho_{k} A-B$ is singular, then solve $\left(\rho_{k} A-B\right) v_{k+1}=0$ to get an approximation $\left(\rho_{k}, v_{k+1}\right)$ and stop; otherwise

(iv) solve $\left(\rho_{k} A-B\right) y_{k+1}=A x_{k}$;

(v) normalise $y_{k+1}$ to get $x_{k+1}=y_{k+1} /\left\|A y_{k+1}\right\|$.

Obviously, difficulties can arise from the fact that $A$ is indefinite. If there is some $k$ such that $x_{k}^{*} A x_{k}=0$, the iteration will break down and no information is obtained. Also, even if $x_{k}^{*} A x_{k} \neq 0$ but oscillates between positive and negative as $k$ increases, there is no way to ensure convergence. Fortunately, it can be shown that these cases will not affect the local convergence to a simple real eigenvalue, and will not happen even for global strategies when the pencil is definite. 
Ostrowski [21] has discussed a generalised Rayleigh quotient method for general non-symmetric matrices (in a so-called two-sided iteration). It is not hard to see that Algorithm 5.1 is equivalent to applying the generalised Rayleigh quotient method to $A^{-1} B$ with a special choice of initial vector pair, that is by taking $\xi_{0}=x_{0}$ and $\zeta_{0}=$ $A x_{0}$ in two-sided iteration. Under some conditions, (with $A$ diagonable, for example) Ostrowski proved that the sequence of Rayleigh quotients $\left\{\rho_{k}\right\}$ converges cubically if it converges to an eigenvalue. But there has been no discussion about the convergence of Rayleigh iterative vectors. A new result of this kind is described here.

Let $\lambda A-B$ be an hermitian pencil with $A$ invertible, and

$$
X^{-1}\left(A^{-1} B\right) X=J \text { or } B X=A X J
$$

where $X=\left[z_{0}, z_{1}, \ldots, z_{n-1}\right]$ is an invertible matrix and $J=\left[\begin{array}{cc}\lambda_{0} & 0 \\ 0 & J_{1}\end{array}\right]$ is a Jordan matrix, with $\lambda_{0}$ a simple real eigenvalue. Let \|\|$_{*}$ be the 2 -norm in the basis $\left\{z_{0}, z_{1}, \ldots, z_{n-1}\right\}$. For the convergence analysis of iterative vectors, we first introduce a measure of convergence. For any $x \in \mathrm{C}^{\mathfrak{n}}$, we have a decomposition

$$
x=a \cdot z_{0}+b \cdot u
$$

where $a, b \in \mathrm{C}$, and $u \in \operatorname{span}\left\{z_{1}, \ldots, z_{n-1}\right\}$ with $\|u\|_{*}=1$. Using this decomposition, for each Rayleigh iteration vector $x_{k}$ of Algorithm 5.1, we can write

$$
x_{k}=a_{k} z_{0}+b_{k} \cdot u_{k} .
$$

Then $c_{k}=\left|b_{k} / a_{k}\right|$ is a measure of how $x_{k}$ approaches $z_{0}$ in direction. Notice that $c_{k}$ only depends on the direction of $x_{k}$.

The following theorem establishes local cubic convergence for the sequence of approximate eigenvectors. The conclusions provide insights into this phenomenon and are not intended to provide computable estimates (see [17]).

Theorem 5.2. Let $\lambda A-B$ be an hermitian matrix pencil with a simple real eigenvalue $\lambda_{0}$ and associated eigenvector $z_{0}$. Let $\left\{x_{k}\right\}_{k=0}^{\infty}$ be defined as in Algorithm 5.1 and $c_{k}$ be defined as above. Then if $c_{0}$ is sufficiently small and $z_{0}$ is A-positive (or $A$-negative) then for all $k, x_{k}$ is $A$-positive (or $A$-negative, respectively). Furthermore, $c_{k+1} \leqslant c_{k}^{3} M$ for some constant $M$ where $k=0,1,2, \ldots$

For the analysis of global convergence properties the available results are still confined to pencils with $A$ positive definite. In the following, this property is generalised to definite pencils. From the point of view of numerical analysis of definite pencils it should be remarked that an alternative strategy is available. Namely, to first compute real numbers $\alpha$ and $\beta$ such that $M:=\alpha A+\beta B>0$, then make a transformation of the 
parameter to create a pencil with positive definite leading term and apply more familiar algorithms to this problem. There are two major reasons for not doing this. First, the computation of $\alpha$ and $\beta$ is itself a substantial task, so that computational efficiency (at the present state of knowledge) does not give clear guidance on the preferred line of attack. Second, the resolution of the direct approach for definite pencils may give some clues on the development of algorithms for indefinite pencils. Furthermore, the results obtained show that the only significant advantage to be gained from making such a shift will be the improved stability resulting from a definite leading coefficient. By working directly with $\lambda A-B$, without a preliminary shift, there may also be some advantage in the freedom to decide whether positive or negative type eigenvalues are to be computed (after transformation of the parameter this distinction is lost).

Note also that if Algorithm 5.1 is applied to the transformed pencil $\lambda M-B$, and to the original pencil $\lambda A-B$ two different recursions are obtained. Thus, different sequences would be obtained from the same starting vectors. Since $M>0$, analysis for the sequence generated by $\lambda M-B$ is an easy extension of the standard results. The presence of $A$ in the recursion generated by $\lambda A-B$ demands the introduction of an indefinite scalar product and some new ideas for the generalisation of these standard results.

The following interesting result shows that the Rayleigh quotient iteration has an $A$-sign invariant property (see [17]).

Theorem 5.3. Let $\lambda A-B$ be a definite pencil with $A$ invertible and $x$ a vector for which $x^{*} A x \neq 0$ and $\rho(x)=\left(x^{*} B x\right) /\left(x^{*} A x\right) \notin \sigma\left(A^{-1} B\right)$. Then for $y=$ $(\rho(x) A-B)^{-1} A x$,

$$
\left(x^{*} A x\right)\left(y^{*} A y\right)>0 \text {. }
$$

A difficulty in the extension of the argument of Kahan and Parlett leading to global convergence results (see $[\mathbf{2 6}, \mathbf{2 4}]$ ), concerns an appropriate choice of norm. When $A>0$ the norm $\left(x, A^{-1} x\right)^{1 / 2}$ is used. When $A$ is indefinite but $\lambda A-B$ is a definite pencil with $M=\alpha A+\beta B>0$, it turns out that the norm $\left(x, M^{-1} x\right)^{1 / 2}$ is an appropriate choice. Using this, the critical monotonicity property of residuals can be established and used to obtain the following result (see [17]).

Theorem 5.4. (Global Convergence Theorem) Let $\lambda A-B$ be an $n \times n$ definite pencil with $n$ distinct eigenvalues, $A$ invertible, and $M=\alpha A+\beta B>0$. Let $\left\{\rho_{k}, x_{k}\right\}$ be the Rayleigh sequence defined by Algorithm 5.1 and the $M^{-1}$-norm. Then

1. $\left\{\rho_{k}\right\}$ converges, and either

2. $\left(\rho_{k}, x_{k}\right) \rightarrow(\lambda, z)$ asymptotically cubically, where $B z=\lambda A z$, or

3. $x_{2 k} \rightarrow x_{+}, x_{2 k+1} \rightarrow x_{-}$, linearly, where $x_{+}$and $x_{-}$are the bisectors of a pair of eigenvectors whose eigenvalues have mean $\rho=\lim _{k} \rho_{k}$. This 
situation is unstable under perturbations of $x_{k}$.

\section{RAYLEIGH-RITZ PROJECTION METhOdS USING KRYLOV SUBSPACES}

Given a self-adjoint operator $A$ acting on a Hilbert space, the idea behind the Rayleigh-Ritz method is to take the compression of $A$ to a subspace, usually with finite dimensions, and use the eigenvalues of the restricted operator as approximations to eigenvalues of $\boldsymbol{A}$. Theoretically, we can take a sequence of subspaces that approaches the whole space in an appropriate sense, and for which the eigenvalues of restricted operators will approach those of $A$ that we want. However, numerically, we must terminate this infinite process at some finite stage. From this point of view, the crucial point is how to choose a good sequence of subspaces that will justify the finite process.

Krylov subspaces $K^{m}(q)$ are candidates and are defined by

$$
K^{m}(q)=\operatorname{span}\left\{q, A q, \ldots, A^{m-1} q\right\}
$$

where $m$ is a positive integer. It is easy to see that $K^{\infty}(q)=\operatorname{span}\left\{q, A q, \ldots, A^{j} q, \ldots\right\}$ is an invariant subspace of $A$. Indeed, it is the smallest $A$-invariant subspace containing $q$. If $A$ is an $n \times n$ matrix, then $K^{n}(q)=K^{\infty}(q)$ is $A$-invariant, but we usually consider Krylov subspaces $K^{m}(q)$ with $m<<n$.

The Rayleigh-Ritz methods using the compression to a Krylov subspace has been very successful for symmetric matrices (for example, Lanczos algorithms, see [22, 23]). One reason for this is the existence of favourable approximation bounds which ensure that a Krylov subspace with relatively low dimension can yield high precision approximations of eigenpairs. We now discuss the formulation of Rayleigh-Ritz methods for symmetric matrix pencil problems.

Let $S$ be a subspace with a basis $\left\{p_{1}, \ldots, p_{m}\right\}$ and denote $P=\left[p_{1}, \ldots, p_{m}\right]$. We define $\lambda A_{1}-B_{1}$ to be a compression pencil of a symmetric pencil $\lambda A-B$, where

$$
A_{1}=P^{*} A P, \quad B_{1}=P^{*} B P .
$$

This compression depends on the choice of the basis. However, the spectrum is invariant under the choice of basis for $S$. In particular, if the basis is orthonormal, then $A_{1}$ and $B_{1}$ are the orthogonal compressions of $A$ and $B$ on $S$ respectively.

For a single matrix, there is an approximation error bound of Kanial-Paige-Sadd and their analysis depends on the classical minimax principle ([13, 22, 29]). Using the minimax principle described above, we are able to extend the analysis to the definite pencil problem. The results obtained provide a theoretical basis for applying projection methods (for example, the generalised symmetric Lanczos algorithms and Arnoldi algorithms) to definite symmetric pencils. 
The main theorem, applied to definite pencils with projection from a Krylov subspace is as follows (see [18]). The importance of this theorem emerges when specific choices of the polynomials $\pi$ are made to determine explicit bounds for the eigenvalues of the compression.

THEOREM 6.1 . Let $\lambda_{r+1} \leqslant \ldots \leqslant \lambda_{n}<\lambda_{1} \leqslant \ldots \leqslant \lambda_{r}$ be the eigenvalues of a definite pencil $\lambda A-B$ and $x_{r+1}, \ldots, x_{n}, x_{1}, \ldots x_{r}$ be corresponding eigenvectors with $x_{i}^{*} A x_{i}=1$ for $i=1, \ldots r$, (that is, $\lambda_{1}, \ldots, \lambda_{r}$ are of positive type) and $x_{i}^{*} A x_{i}=-1$ for $i=r+1, \ldots, n$ (that is, $\lambda_{r+1}, \ldots, \lambda_{n}$ are of negative type). Let $\theta_{1} \leqslant \ldots \leqslant \theta_{p}$ be the positive type eigenvalues of $\lambda A_{1}-B_{1}$, the compression of $\lambda A-B$ on a Krylov subspace $K^{m}(q)$, and $z_{1}, \ldots, z_{p} \in \mathrm{C}^{m}$ be corresponding eigenvectors. Then $p \leqslant r$. Furthermore, if $q=\sum_{i=1}^{n} \alpha_{i} x_{i}$ and we define

$$
a_{k}(\pi)=\sum_{i=1}^{n}\left|\pi\left(\lambda_{i}\right) \alpha_{i}\right|^{2}\left(\lambda_{i}-\theta_{i}\right)^{2} \ldots\left(\lambda_{i}-\theta_{p}\right)^{2} \operatorname{sgn}\left(x_{i}^{*} A x_{i}\right)
$$

for any polynomial $\pi$, then for $k=1, \ldots, p$

$$
\lambda_{k} \leqslant \theta_{k} \leqslant \lambda_{k}+\varepsilon_{k}
$$

where $\quad \varepsilon_{k}=\min _{\substack{\pi \in P^{m-k} \\ a_{k}(\pi)>0}} \sum_{i=k+1}^{n} \frac{\left|\pi\left(\lambda_{i}\right) \alpha_{i}\right|^{2}\left(\lambda_{i}-\theta_{i}\right)^{2} \ldots\left(\lambda_{i}-\theta_{p}\right)^{2}\left|\lambda_{i}-\lambda_{k}\right|}{a_{k}(\pi)}$.

This expression suggests that we expect smaller positive type eigenvalues (that is, $\lambda_{1}, \lambda_{2}$ et cetera) to converge first. It is observed in [7] and [25] that in Lanczos algorithm the bigger and smaller eigenvalues of the whole spectrum converge first as in the classical case. Our numerical experience with Lanczos algorithms shows that for large pencils with good separation of the two eigenvalue types, the larger and smaller eigenvalues of both positive and negative types converge first.

We can bound $\varepsilon_{k}$ by choosing an appropriate polynomial $\pi \in P^{m-k}$ in the minimisation functional. Let $T_{l}$ denote the Chebyshev polynomial of degree $l$ with fundamental domain $[-1,1]$. Then the following polynomial of degree $2 l$

$$
P_{l, a}(x)=T_{l}\left[\frac{2 x^{2}-a^{2}-1}{a^{2}-1}\right]
$$

has the property that it is bounded in the intervals $[-a,-1]$ and $[1, a]$ by 1 , and increases rapidly as $x$ approaches zero. By a transformation of intervals, we obtain a polynomial that is large at $\lambda_{k}$ and bounded by 1 in $\left[\lambda_{k+1}, \lambda_{r}\right]$ and $\left[\lambda_{r+1}, \lambda_{n}\right]$. We therefore apply this polynomial to get a bound for $\varepsilon_{k}$. The details can be found in [18]. 


\section{LANCZOS ALgORITHMS FOR $\lambda A-B$}

As a special Rayleigh-Ritz projection method, the method of Lanczos generates a sequence of orthogonal bases for Krylov subspaces via a three term recurrence. Its favourable approximation property has made it a popular method for large symmetric matrices when only a few eigenvalues are needed (see $[22,23]$ for the classical case). For $\lambda A-B$ with $A$ positive definite, the same idea can be applied to construct an $A$ orthogonal basis for a Krylov subspace and it is equivalent to application of the method to a symmetric matrix. In our case $A$ is merely invertible, but a similar three term recurrence can be used to generate bases for Krylov subspaces, which are orthogonal with respect to the indefinite scalar product defined by $A$. We formulate the algorithm as follows:

ALgORIthM 7.1. Given an initial vector $q_{1}$ with $\varepsilon_{1}=q_{1}^{*} A q_{1}= \pm 1$ and $\alpha_{1}=q_{1}^{*} B q_{1}$, the Lanczos algorithm generates $\left\{q_{1}, q_{2}, \ldots, q_{m}\right\},\left\{\alpha_{1}, \alpha_{2}, \ldots, \alpha_{m}\right\}$, $\left\{\beta_{1}, \beta_{2}, \ldots, \beta_{m-1}\right\}$ and $\left\{\varepsilon_{1}, \varepsilon_{2}, \ldots, \varepsilon_{m}\right\}$ by the following recursion. Denote $\beta_{0} q_{0}=0$ and $\varepsilon_{0}=0$, then for $j=1,2, \ldots$

1. $r_{j}=\left(A^{-1} B\right) q_{j}-\varepsilon_{j-1} \beta_{j-1} q_{j-1}-\varepsilon_{j} \alpha_{j} q_{j}$,

2. if $\boldsymbol{r}_{j}=0$, stop; otherwise,

3. if $r_{j}^{*} A r_{j}=0$, stop; otherwise,

4. $\beta_{j}=\sqrt{\left|r_{j}^{*} A r_{j}\right|}, \quad \varepsilon_{j+1}=\operatorname{sgn}\left(r_{j}^{*} A r_{j}\right), \quad q_{j+1}=r_{j} / \beta_{j} \varepsilon_{j+1}$ and $\alpha_{j+1}=q_{j+1}^{*} B q_{j+1}$.

If there is no break down in generating $\left\{q_{1}, \ldots, q_{j}\right\}$ (that is, the cases 2 and 3 do not happen), it is easy to check that for $Q_{j}=\left[q_{1}, \ldots, q_{j}\right]$ we have

$$
A^{-1} B Q_{j}=Q_{j} P_{j} T_{j}+r_{j} e_{j}^{*}
$$

and

$$
Q_{j}^{*} A Q_{j}=P_{j}
$$

where $e_{j}^{*}=(0, \ldots, 0,1) \in \mathbf{R}^{j}, P_{j}=\operatorname{diag}\left(\varepsilon_{1}, \ldots, \varepsilon_{j}\right)$ and

$$
T_{j}=\left[\begin{array}{rrr}
\alpha_{1} \beta_{2} & & \\
\beta_{2} \alpha_{2} & & \\
& \ddots & \\
& & \beta_{j} \\
& & \beta_{j} \alpha_{j}
\end{array}\right]
$$

is tridiagonal. In this algorithm, we normalise $q_{j}$ so that $\left|q_{j}^{*} A q_{j}\right|=1$ as in the case when $A$ is positive definite. But $r_{j}^{*} A r_{j}$ could be very small (since $A$ is indefinite) and 
hence $\left\|q_{j}\right\|$ could be very large. For this reason we introduce another normalisation, namely, $\left\|q_{j}\right\|=1$. Another advantage of this normalisation is that $q_{j}^{*} A q_{j}$ provides us with a criterion for when the algorithm breaks down. In this normalisation, we must modify equation (7.1), and the $\varepsilon_{j}$ there would no longer be \pm 1 but a non-zero number. The details will not be presented here but referred to [25].

Now, after the $j$-step of the Lanczos algorithm has been preformed, we have $T_{j}=$ $Q_{j}^{*} B Q_{j}$. Thus we obtain a triangular compression pencil $\lambda P_{j}-T_{j}=Q_{j}^{*}(\lambda A-B) Q_{j}$.

If $\theta_{i}$ and $s_{i}=\left(s_{1 i}, \ldots, s_{j i}\right)^{T}$ are an eigenvalue and the corresponding eigenvector of $\lambda P_{j}-T_{j}$, then $\left(\theta_{i}, y_{i}\right)$ with $y_{i}=Q_{j} s_{i}$ is called a Ritz pair and is used as an approximation of an eigenpair of $\lambda A-B$. The approximation can be measured by the residual of the Ritz pair and the following identity concerning the residuals of Ritz pairs is easily proved:

$$
B y_{i}-\theta_{i} A y_{i}=s_{j i} A\left(\varepsilon_{j+1} \beta_{j+1} q_{j+1}\right)
$$

So, an $s_{j i}$ of small absolute value signals the convergence of Ritz pair $\left(\theta_{i}, y_{i}\right)$, provided $\beta_{j+1} A q_{j+1}$ is bounded. However, in general the error of eigenvalue approximation cannot be bounded by this residual. This is due to the non-symmetric nature of the problem. One way to overcome this difficulty is to relate the residual to a perturbation $E$ of $B$. It has been proved in [12] and [25] that $\left(\theta_{i}, y_{i}\right)$ is an eigenpair of $\lambda A-(B+E)$ with $\|E\|=O\left(s_{j i}\right)$. From this, $\left|\lambda_{i}-\theta_{i}\right|=O\left(s_{j i}\right)$ for some eigenvalue $\lambda_{i}$. In [18], we use the tridiagonal structure of Algorithm 7.1 rather than the identity (7.2) and under some moderate conditions, it is shown that if $s_{j i}$ is small enough

$$
\lambda_{i}-\theta_{i}=\gamma s_{j i}^{2}+O\left(s_{j i}^{3}\right)
$$

where $\gamma$ is a constant. Unfortunately, $\gamma$ is not computable in this expression.

For definite pencils, the following result gives a direct bound on $\min _{l}\left|\theta_{i}-\lambda_{l}\right|$ by $s_{j i}$ and extends the classical case (see [18]).

THEOREM 7.2. Let $\left\{\lambda_{i}\right\}$ be the eigenvalues of a definite pencil $\lambda A-B$ with $A$ invertible and $M=\alpha A+\beta B>0$. If $\left(\theta_{i}, y_{i}\right)$ is a Ritz pair, then

$$
\min _{l}\left|\theta_{i}-\lambda_{l}\right| \leqslant \frac{\max _{l}\left|\alpha+\beta \lambda_{l}\right|}{\min _{l} \sqrt{\left|\alpha+\beta \lambda_{l}\right|}} s_{j i}\left\|\beta_{j+1} A q_{j+1}\right\|_{M^{-1}} .
$$

Since this bound is invariant under scaling of $\alpha$ and $\beta$, there is no reason to expect that the term on the right-hand-side is very much bigger than $s_{j i}\left\|\beta_{j+1} A q_{j+1}\right\|_{M^{-1}}$. Of course, these analyses are not intended to provide a computable bound, but shows that a small $s_{j i}$ does indeed signal the convergence of a Ritz pair. 


\section{REFERENCES}

[1] C. Beattie and D.W. Fox, 'Localization criteria and containment for Rayleigh quotient iteration'; SIAM J. Matrix Anal. Appl. 10 (1989), 80-93.

[2] N. Burgoyne and R. Cushman, 'Normal forms for real linear Hamiltonian systems with purely imaginary eigenvalues', Celestial Mech. 8 (1974), 435-443.

[3] R. Courant, 'Uber die Eigenwerte bei den Differentialgleichungen der mathematischen Physik', Math Z. 7 (1920), 1-57.

[4] S.H. Crandall, 'Iterative procedures related to relaxation methods for eigenvalue problems', Proc. Roy. Soc. London Ser. A 207 (1951), 416-423.

[5] C.R. Crawford, 'A stable generalized eigenvalue problem', SIAM J. Numer. Anal. 6 (1976), 854-860.

[6] R.J. Duffin, 'A minimax theory for overdamped networks', Arch. Rational Mech. Anal. 4 (1955), 221-233.

[7] T. Ericsson and A. Ruhe, 'Lanczos algorithm and field of value rotations for symmetric matrix pencils', Linear Algebra Appl. 88/89 (1987), 733-746.

[8] E. Fischer, 'Uber quadratische Formen mit reellen Koeffizienten', Monatsh. Math. 16 (1905), 234-409.

[9] I. Gohberg, P. Lancaster and L. Rodman, Matrices and Indefinite Scalar Products (Birkhäuser, Basel, 1983).

[10] D.J. Inman, Vibration (Prentice Hall, Englewood Cliffs, 1989).

[11] W. Kahan, 'Inclusion theorems for clusters of eigenvalues of hermitian matrices', in Tech nical Report (Dept. of Comp. Sci., University of Toronto, 1967).

[12] W. Kahan, B. Parlett and E. Jiang, 'Residual bounds on approximate eigensystems of nonnormal matrices', SIAM J. Numer. Anal. 19 (1982), 470-484.

[13] S. Kaniel, 'Estimates for some computational techniques in linear algebra', Math. Comp. 20 (1966), 369-378.

[14] P. Lancaster, 'A generalized Rayleigh quotient iteration for lambda-matrices', Arch. Rational Mech. Anal. 8 (1961), 309-322.

[15] A. Laub and K. Meyer, 'Canonical forms for sympletic and Hamiltonian matrices', Celestial Mechanics 9 (1974), 213-238.

[16] P. Lancaster and Q. Ye, 'Inverse spectral problems for linear and quadratic matrix pencils', Linear Alg. Appl. 107 (1988), 293-309.

[17] P. Lancaster and Q. Ye, 'Variational properties and Rayleigh quotient algorithms for symmetric matrix pencils', in Operator Theory: Advances and Applications 40, pp. 247-278 (Birkhauser, Basel, 1989).

[18] P. Lancaster and Q. Ye, 'Rayleigh-Ritz and Lanczos methods for symmetric matrix pencils', (preprint).

[19] P. Lancaster and M. Tismentsky, The Theory of Matrices (Academic Press, Orlando, 1985).

[20] B. Najman and $Q$. Ye, 'A minimax characterization for eigenvalues of hermitian pencils', Linear Algebra Appl. (to appear). 
[21] A Ostrowski, 'On the convergence of the Rayleigh quotient iterstion for the computation of characteristic roots and vectors', I-VI, Arch. Rational Mech. Anal. 1-4 (1958/1959), 233-241, 423-428, 325-340, 341-347, 472-481, 153-165.

[22] C. Paige, The computation of eigenvalues and eigenvectors of very large sparse matrices, Ph.D. dissertation (University of London, 1971).

[23] B.N. Parlett, The Symmetric Eigenvalue Problem (Prentice-Hall, Englewood Cliffs, N.J., 1980).

[24] B.N. Parlett, 'The Rayleigh quotient iteration and some generalizations for non-normal matrices', Math. Comp. 28 (1974), 679-693.

[25] B.N. Parlett and H. Chen, 'Use of an indefinite inner product for computing damped natural modes', (preprint).

[26] B.N. Parlett and W. Kahan, 'On the convergence of a practical $Q R$ algorithm', Information Processing 68, I Mathematics, Software, pp. 114-118, (North-Holland, Amsterdam, 1969).

[27] R.S. Phillips, 'A minimax characterization for the eigenvalues of a positive symmetric operator in a space with an indefinite metric', J. Fac. Sci. Univ. Tokyo Sect. IA Math. 17 (1970), 51-59.

[28] E.H. Rogers, 'A minimax theory for overdamped systems', Arch. Rational Mech. Anal. 16 (1964), 89-96.

[29] Y. Saad, 'On the rate of convergence of the Lanczos and block Lanczos methods', SIAM J. Numer. Anal. 17 (1980), 687-706.

[30] G.W. Stewart, 'Perturbation bounds for the definite generalized eigenvalue problem', Linear Alg. Appl. 23 (1979), 69-85.

[31] G. Temple, 'The accuracy of Rayleigh's method of calculating the natural frequencies of vibrating systems', Proc. Roy. Soc. London 211 (1952), 204-224.

[32] B. Textorius, 'Minimaxprinzipe zur Bestimmung der Eigenwerte $J$-nichtnegativer Operatoren', Math. Scand. 35 (1974), 105-114.

[33] R. Turner, 'Some variational principles for a nonlinear eigenvlaue problem', J. Math. Anal. Appl. 17 (1967), 151-165.

[34] P. Van Dooren and P. Dewilde, 'The eigenstructure of an arbitrary polynomial matrix: computational aspects', Linear Algebra Appl. 50 (1983), 545-579.

[35] K. Weierstrass, 'Zur Theorie der bilinearen und quadratischen Formen', Monatsber. Akad. Wiss. Berl. (1868), p. 310.

[36] Q. Ye, Variational principles and numerical algorithms for symmetric matrix pencils, Ph.D. Thesis (University of Calgary, 1989).

Department of Mathematics and Statistics

University of Calgary

Calgary, Alberta

Canada T2N 1N4 